\title{
Zoom y Moodle: acortando distancias entre universidades. Una experiencia entre la Universidad de Guadalajara, México y la Universidad Libre de Colombia
}

\section{Cristian Chiñas-Palacios ${ }^{\mathrm{a}}$, Carlos Vargas-Salgado ${ }^{\mathrm{b}}$, Jesús Águila-León ${ }^{\mathrm{c}}$, Edith X. M. García $^{\mathbf{d}}$}

a,c,dDepartamento de Estudios del Agua y la Energía, Universidad de Guadalajara Centro Universitario de Tonalá, Jalisco, México.

${ }^{b}$ Departament d'Enginyeria Elèctrica, Universitat Politècnica de València, València, España.

adaniel.chinas@academicos.udg.mx, ${ }^{b}$ carvarsa@upvne.upv.es, cjesus.aguila@academicos.udg.mx, dxio.garcía@academicos.udg.mx

\begin{abstract}
This paper presents the use of the Moodle platform and the application of Zoom as part of the Information and Computing Technologies for the development of an International Diploma in Renewable Energy carried out between the University of Guadalajara, Mexico and the University Free of Colombia. The design of the diploma, the thematic modules and the distribution of face-to-face and non-face-to-face sessions are described. Subsequently, the average grades obtained by the students for the non-face-toface and face-to-face sessions are analyzed. The results show that the use of a virtual environment for course management in conjunction with an interactive videoconferencing platform, such as Moodle and Zoom, help the student obtain better grades by achieving better retention and learning of the content of the sessions.
\end{abstract}

Keywords: Videoconference, Virtual Environment, Zoom, Moodle, International, Remote sessions, Renewable Energies.

\section{Resumen}

En este trabajo se presenta un método de enseñanzalaprendizaje a través de la plataforma Moodle y la aplicación de Zoom como recursos tecnológicos para la docencia. Esta técnica se aplica al desarrollo de un Diplomado Internacional en Energías Renovables llevado a cabo entre la Universidad de Guadalajara, México y la Universidad Libre de Colombia. Se describe el diseño del diplomado, los módulos temáticos y la distribución de sesiones presenciales y no presenciales. Posteriormente se comparan y analizan las notas promedio obtenidas por los estudiantes para las sesiones nopresenciales y presenciales. Los resultados muestran que el empleo de un entorno virtual, mediante la aplicación de las tecnologías de la Información y la Computación (TIC) para gestión de cursos, en conjunto con una plataforma interactiva de videoconferencias, como lo son Moodle y Zoom, ayudan al estudiante a asimilar mejor los conceptos vistos en la asignatura. 
Zoom y Moodle: acortando distancias entre universidades. Una experiencia entre la Universidad de Guadalajara, México y la Universidad Libre de Colombia.

Palabras clave: Videoconferencia, Entorno Virtual, Zoom, Moodle, Internacional, No-presencial, Energías Renovables, método de enseñanza/aprendizaje.

\section{Introducción}

La educación a distancia ha sido en los países Iberoamericanos una de las principales estrategias para la difusión del conocimiento y la formación de alumnos entre comunidades lejanas (Maher \& Prescott, 2017), además de abrir una oportunidad para poder realizar cursos y talleres de formación profesional a distancia (Vesisenaho, Valtonen, Wulff, \& Kuittinen, 2019) .

Muchas universidades han hecho esfuerzos por emplear innovaciones en el aula mediante entornos virtuales que favorezcan el proceso de enseñanza-aprendizaje, tales como repositorios multimedia para presentaciones de clases, conferencias, talleres como en el caso de la Universitat Politècnica de València (UPV) con la plataforma institucional de RiuNet (https://riunet.upv.es/), aunque su utilización no está muy extendida aún entre la comunidad universitaria (De Andrés Martínez et al., 2018); también han tratado de llevar el aula más allá de sus límites físicos al emplear gestores de clase virtuales donde el docente puede crear múltiples grupos para diversas asignaturas, dar de alta alumnos, dejar tareas, evaluar, enviar avisos como lo es Google Classroom (Heggart \& Yoo, 2018), Moodle (Oproiu, 2015) y PoliformaT de la UPV (UPV, 2018) e incluso realizar evaluaciones (Grison, 2018) de manera dinámica por medio de la gamificación y a través de videoconferencias para la oferta de cursos a distancia (Sutterlin, 2019) con recursos tecnológicos para la docencia que permitan una mayor interacción entre los alumnos y el profesor (Blau, Weiser, \& Eshet-Alkalai, 2016), como lo es Zoom. Esta herramienta favorece las relaciones a distancia a través de reuniones en línea para conferencia, talleres, seminarios o eventos de colaboración entre distintas instituciones u organizaciones (Lenkaitis, 2019). Zoom posee características importantes sobre sus demás competidores, permitiendo el acceso a una reunión a través de un código generado en la aplicación y sin necesidad de haber creado una cuenta de usuario previo para acceder a la videoconferencia, como suele ser el caso de otros servicios similares. La Figura 1 muestra la ventana principal al acceder a la página web de Zoom (www.zoom.us). 


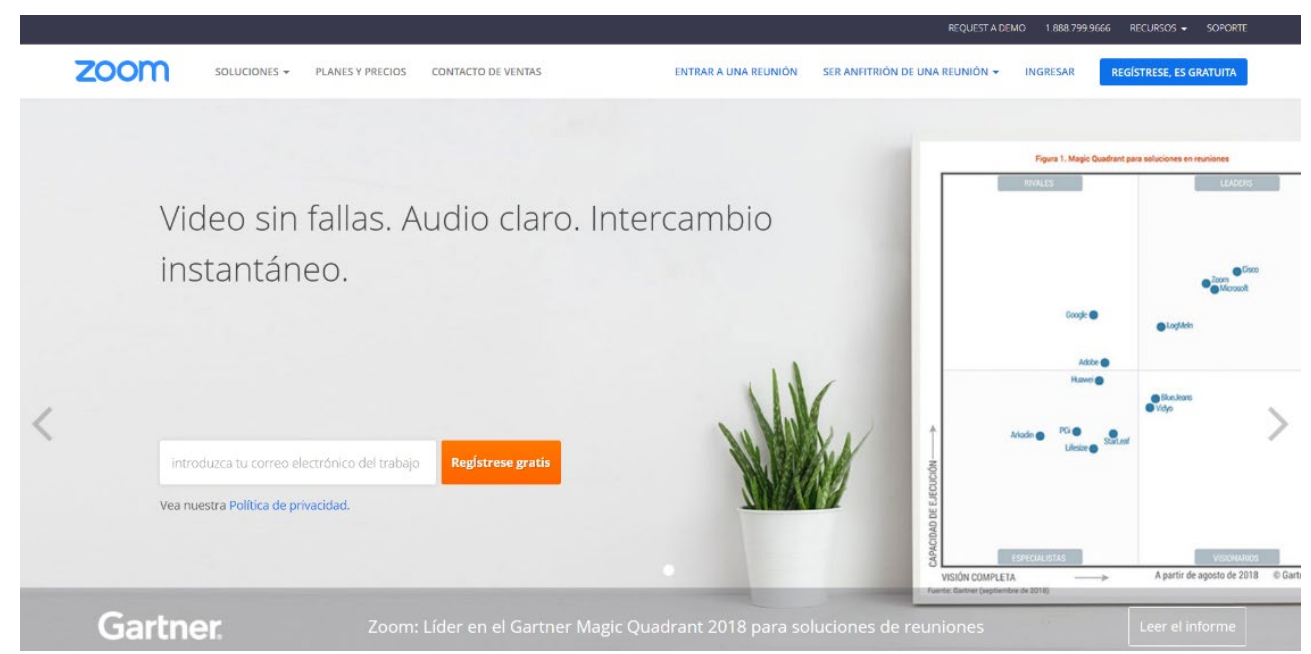

Fig. 1 Ventana principal de la página de Internet de la plataforma Zoom.

En el marco de la implementación del Diplomado Internacional en Energías Renovables surgido a raíz de un convenio existente entre la Universidad Libre de Colombia, Colombia, y la Universidad de Guadalajara, México, se presenta en este trabajo una descripción general de dicho diplomado, la experiencia y los resultados obtenidos por la implementación de plataformas y repositorios virtuales, destacando el empleo de la herramienta Zoom como medio de videoconferencias para los talleres no presenciales entre ambas universidades y de la plataforma Moodle como repositorio del contenido multimedia del diplomado. La Figura 2 muestra el portal de acceso de Moodle.

\section{(1) CUTONALÁ}

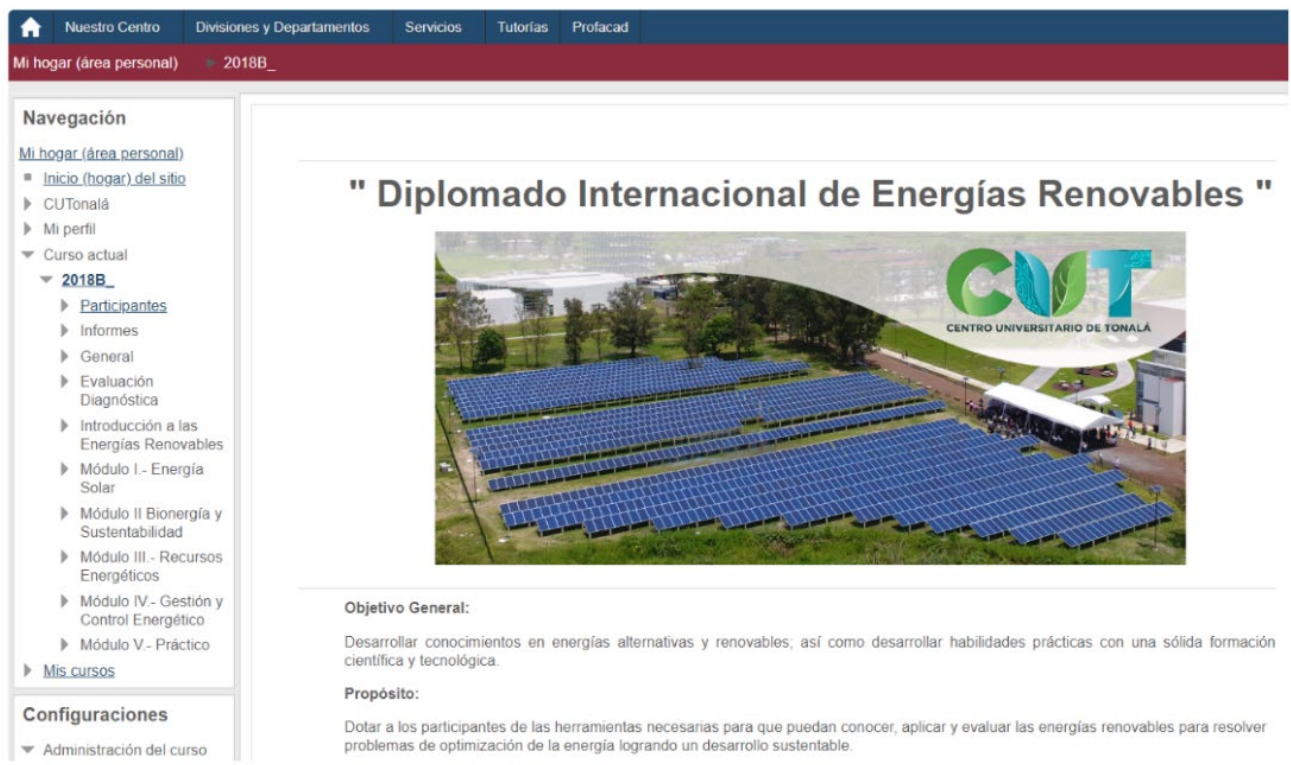

Fig. 2 Página principal del Diplomado en Energías Renovables en Moodle. 


\section{Objetivos}

El objetivo del presente trabajo es dar a conocer el potencial, la experiencia y los resultados obtenidos del empleo de la plataforma de Zoom para enseñanza a distancia integrando Moodle como entorno virtual para el Diplomado Internacional en Energía Renovable llevado a cabo entre la Universidad de Guadalajara, México y la Universidad Libre de Colombia, Colombia, con la finalidad de incentivar el desarrollo de actividades interinstitucionales a distancia.

\section{Desarrollo de la innovación}

\subsection{Descripción y criterios}

La Universidad de Guadalajara y la Universidad Libre de Colombia firmaron un convenio de colaboración para la realización de un Diplomado Internacional en Energías Renovables, gestionado por el Centro Universitario de Tonalá en México, para estudiantes colombianos, con la finalidad de que estudiantes colombianos puedan realizar estudios y matricularse en el diplomado ofrecido por la Universidad de Guadalajara. Mediante esta herramienta el profesor puede autorizar al estudiante de forma virtual. El diplomado tuvo una duración de 150 horas en total.

El diplomado tuvo las siguientes características:

- Se matricularon 25 alumnos, 14 de ellos de la carrera de Ingeniería Ambiental y 11 de la carrera de Ingeniería Mecánica, todos ellos de la Universidad Libre de Colombia.

- El diplomado estuvo gestionado por la Universidad de Guadalajara, por lo que todas las sesiones fueron impartidas por profesores de esta universidad, algunas de ellas de manera presencial y otras de forma virtual según se había planteado en el módulo de aprendizaje.

- Una vez concluido el diplomado los alumnos, en equipos de tres integrantes, tuvieron la oportunidad de elegir a un profesor asesor para su proyecto de fin de grado.

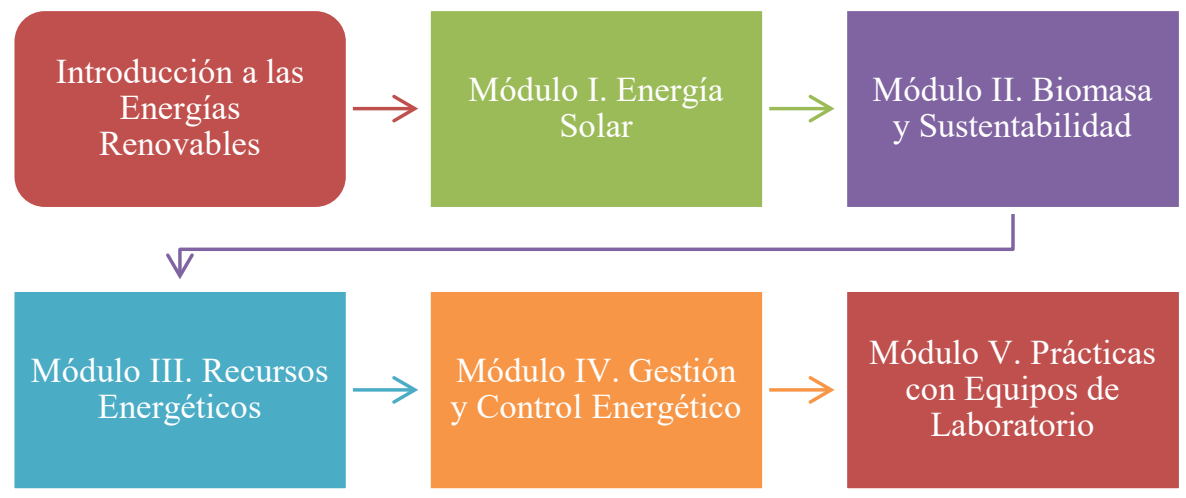

Fig. 3 Módulos de aprendizaje del Diplomado Internacional en Energías Renovables Energía

El diplomado se dividió en 5 módulos de aprendizaje, según puede apreciarse en el esquema de la Figura 3. Además, se dio una introducción a las energías renovables y se realizaron distintas prácticas de laboratorio con material especializado para prácticas energías renovables. 
La Tabla 1 muestra los contenidos temáticos para cada módulo del diplomado, así como el tipo de curso y si es de carácter presencial o no presencial. En total se tuvieron 15 sesiones, 9 de ellas en modalidad no presencial y 6 presenciales.

Tabla 1. Contenidos temáticos de los módulos del Diplomado Internacional en Energías Renovables

\begin{tabular}{|c|c|c|}
\hline Módulo & Tema & Modalidad \\
\hline \multirow[t]{5}{*}{ Energía Solar } & Energía solar y termoconversores solares & No presencial \\
\hline & Tipos de celdas solares & No presencial \\
\hline & Tecnologías de concentración solar & No presencial \\
\hline & $\begin{array}{l}\text { Aplicación de las energías renovables en la } \\
\text { desalación de agua de mar }\end{array}$ & No presencial \\
\hline & $\begin{array}{l}\text { Diseño y optimización de celdas solares } \\
\text { sensibilizadas para la captación de energía renovable }\end{array}$ & No presencial \\
\hline \multirow[t]{2}{*}{ Bioenergía y Sustentabilidad } & Modelos matemáticos para la generación de biogás & Presencial \\
\hline & $\begin{array}{l}\text { Uso del mecanismo celular de microorganismos en la } \\
\text { obtención de energía }\end{array}$ & Presencial \\
\hline \multirow[t]{3}{*}{ Recursos Energéticos } & Energía Eólica & No presencial \\
\hline & $\begin{array}{l}\text { Recursos hídricos y generación hidroeléctrica en el } \\
\text { marco de la sustentabilidad }\end{array}$ & No presencial \\
\hline & Energía geotérmica: aplicaciones y tendencias & No presencial \\
\hline \multirow[t]{4}{*}{ Gestión y Control Energético } & $\begin{array}{l}\text { Tendencias en el desarrollo e implementación de } \\
\text { tecnologías en Energías Renovables }\end{array}$ & Presencial \\
\hline & $\begin{array}{l}\text { Instrumentación y sistemas de control para las } \\
\text { energías renovables }\end{array}$ & Presencial \\
\hline & $\begin{array}{l}\text { Microrredes eléctricas basadas en energías } \\
\text { renovables }\end{array}$ & Presencial \\
\hline & $\begin{array}{l}\text { Sistemas de información con aplicaciones para } \\
\text { energías renovables }\end{array}$ & No presencial \\
\hline \multirow{8}{*}{$\begin{array}{l}\text { Prácticas de Laboratorio de } \\
\text { Energías Renovables }\end{array}$} & Colector plano termosolar & Presencial \\
\hline & Simulador fotovoltaico & \\
\hline & Túnel de viento & \\
\hline & Generador eólico & \\
\hline & Turbinas hidráulicas & \\
\hline & Banco hidráulico & \\
\hline & Microrredes & \\
\hline & Motor Stirling & \\
\hline
\end{tabular}




\subsection{Integración de Moodle a las evaluaciones}

La plataforma de Moodle es una herramienta utilizada para la metodología de enseñanza/aprendizaje. En ella se lleva el control de las tareas, actividades y trabajos de todos los participantes dentro del diplomado. La Figura 4 muestra un esquema de la metodología seguida para el desarrollo de las actividades de cada sesión.

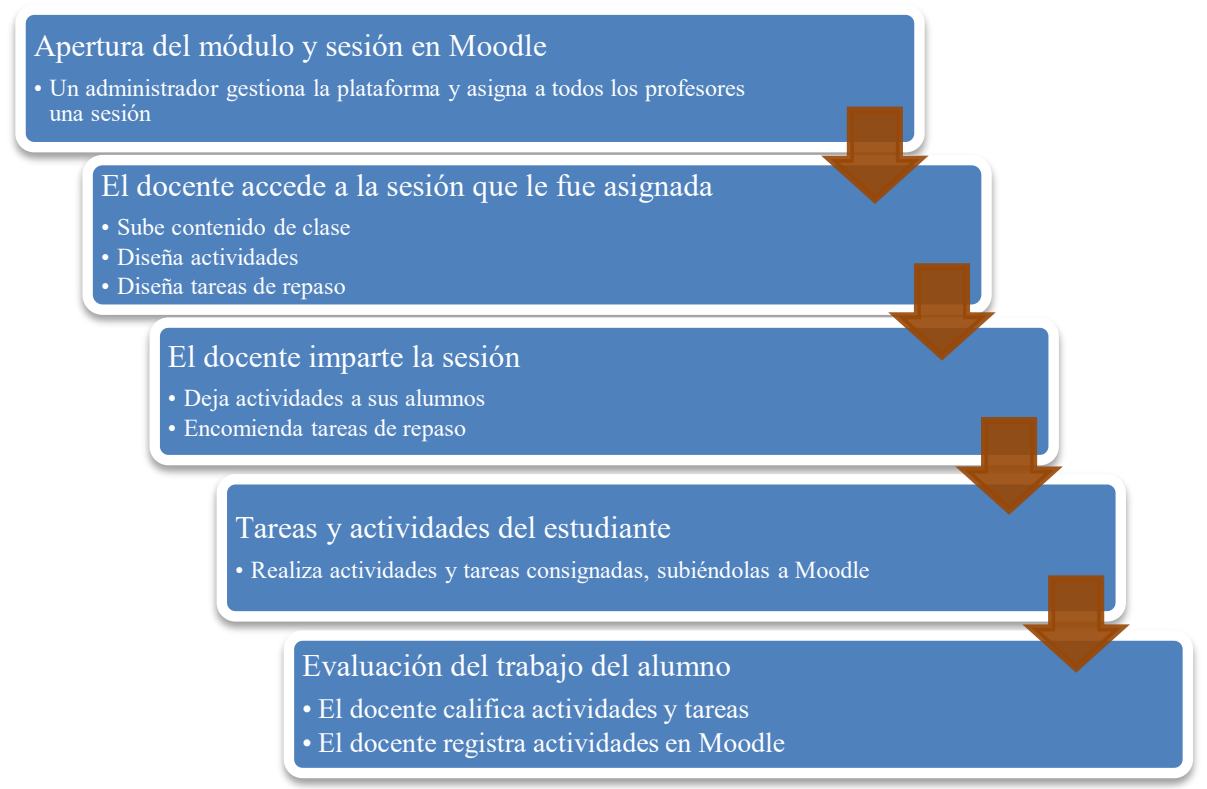

Fig. 4 Metodología del proceso de creación, impartición y evaluación de las sesiones del diplomado

\subsection{Utilización de Zoom para las sesiones no presenciales}

Como se mencionó con anterioridad, algunas de las sesiones del diplomado fueron en modalidad no presencial, estas se llevaron a cabo empleando Zoom como plataforma. Cada docente creó una cuenta y compartió un código de invitación con sus alumnos. Una vez los alumnos se conectaron a la sesión de Zoom, se da inicio a la clase. El profesor puede visualizar en todo momento de forma remota el aula de clases, como muestra la Figura 5. Los videos de las sesiones de las clases impartidas por el profesor se guardan y el estudiante puede acceder a ellos en el momento que lo requiera, funcionando de forma similar a una plataforma MOOC (por sus siglas en inglés, Massive Open Online Courses). 
Grabación_Videoconferencia_Tecnologías de Concentración Solar

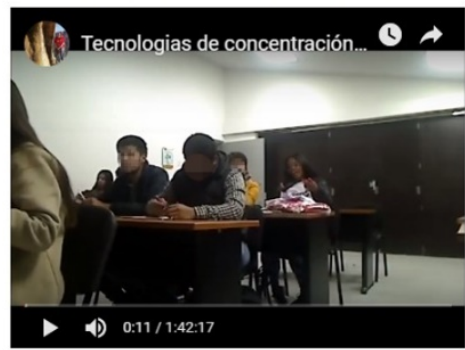

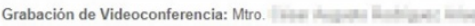

Dia: Jueves 23 de Agosto de $18: 00$ - 20:00 hrs.

Fig. 5 Vista en tiempo real del salón de clases desde el ordenador del profesor

La Figura 6(a) muestra a un docente, en una captura de pantalla, realizando su presentación, y en la Figura 6(b) se aprecia a un alumno realizando una pregunta al profesor. A través de Zoom, los alumnos pueden pedir la palabra para realizar preguntas.

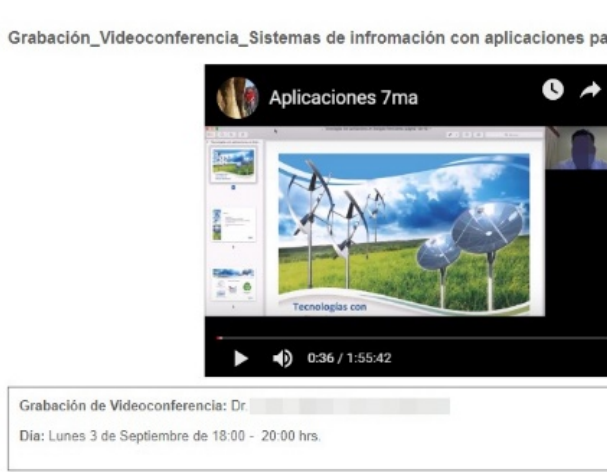

(a)

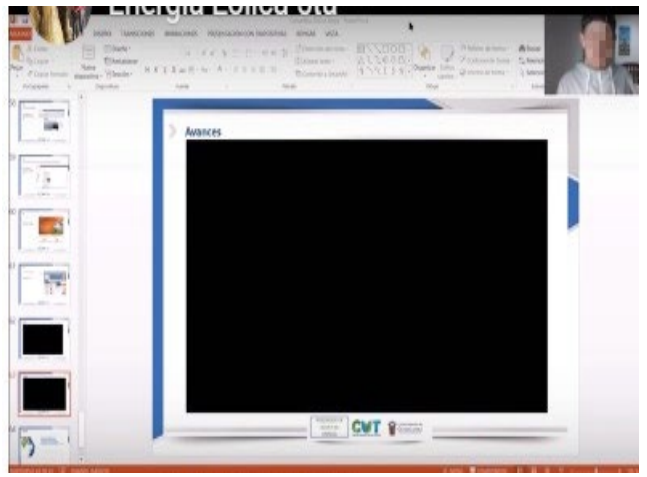

(b)

Fig. 6 (a) Docente realizando exposición a través de Zoom, (b) estudiante realizando una pregunta al profesor durante la exposición

\section{Resultados}

Como resultado de las sesiones presenciales y no presenciales del diplomado se obtuvo una la nota de cada alumno y su análisis, como se muestra en la Figura 7. 


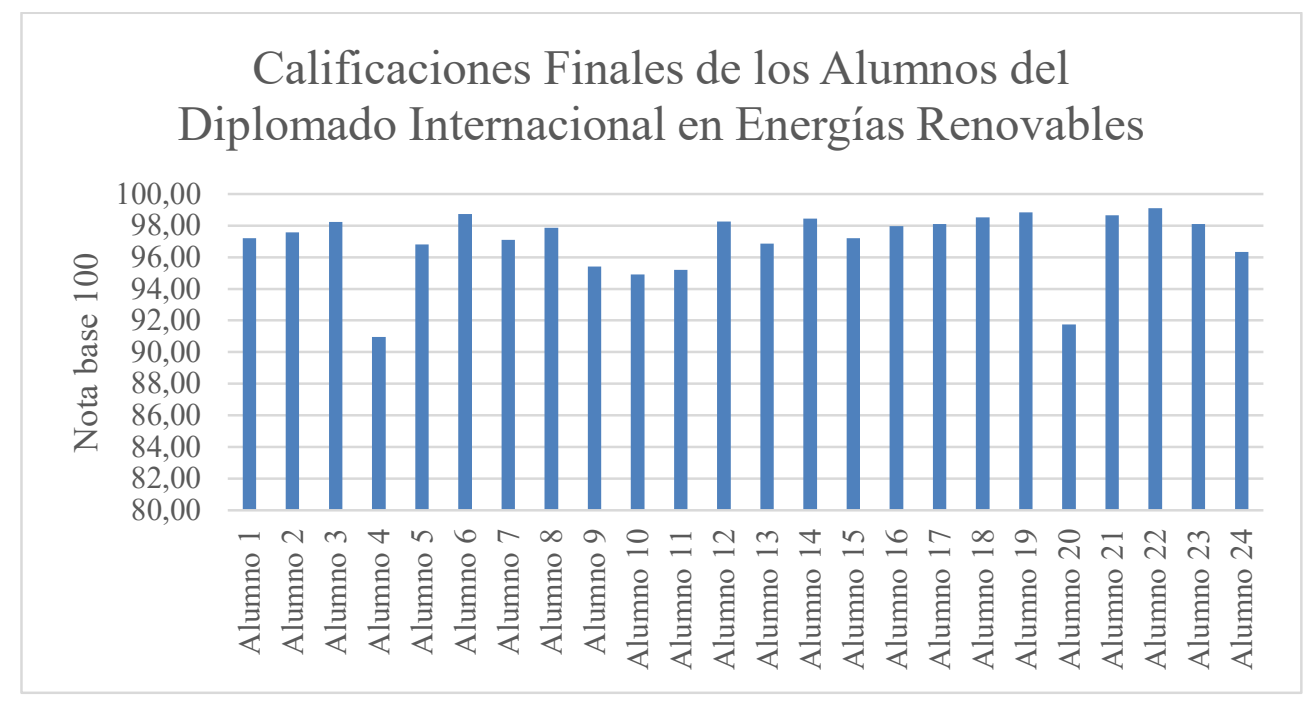

Fig. 7 Calificación final del grupo que tomó el Diplomado Internacional en Energías Renovables

Las notas obtenidas en las sesiones no presenciales del Diplomado Internacional en Energías Renovables fueron comparadas con las notas obtenidas en las sesiones presenciales. Tanto para las sesiones presenciales como las no presenciales se empleó el mismo criterio de evaluación. Adicionalmente se hace un análisis de cuáles fueron las sesiones que obtuvieron una nota más baja y cuáles una más alta.

Tabla 2. Notas obtenidas en las sesiones presenciales y no presenciales del Diplomado Internacional en Energías Renovables

\begin{tabular}{ccccc}
\hline $\begin{array}{c}\text { Nota obtenida (en } \\
\text { base 100) }\end{array}$ & $\begin{array}{c}\text { Sesiones presenciales impartidas en } \\
\text { CUTonalá }\end{array}$ & \multicolumn{2}{c}{ Sesiones no presenciales empleando } \\
Foom & & \multicolumn{2}{c}{ Zomencia } \\
absoluta & $\begin{array}{c}\text { Frecuencia } \\
\text { relativa }\end{array}$ & $\begin{array}{c}\text { Frecuencia } \\
\text { absoluta }\end{array}$ & $\begin{array}{c}\text { Frecuencia } \\
\text { relativa }\end{array}$ \\
\hline $80-90$ & 2 & $8 \%$ & 1 & $4 \%$ \\
$90-92$ & 3 & $13 \%$ & 0 & $0 \%$ \\
$92-94$ & 0 & $0 \%$ & 0 & $0 \%$ \\
$94-96$ & 2 & $8 \%$ & 1 & $4 \%$ \\
$96-97$ & 3 & $13 \%$ & 1 & $4 \%$ \\
$97-98$ & 7 & $29 \%$ & 3 & $13 \%$ \\
$98-99$ & 6 & $25 \%$ & 13 & $54 \%$ \\
$99-100$ & 1 & $4 \%$ & 5 & $21 \%$ \\
\hline
\end{tabular}

La Tabla 2 muestra las frecuencias absolutas y relativas de las notas obtenidas por los estudiantes del Diplomado Internacional de Energías Renovables para las sesiones presenciales y las no presenciales. Se ha de remarcar como el rango de calificaciones 
obtenidas entre 98 a 99 y de 99 a 100 son superiores en un $29 \%$ y $17 \%$, respectivamente, para las sesiones no-presenciales con uso de Zoom en comparación las notas obtenidas por los estudiantes durante las sesiones presenciales tradicionales.

La Fig. 8 representa el porcentaje de la calificación final del diplomado para aquellas clases que se impartieron de manera presencial, en el Centro Universitario de Tonalá, y aquellas que se impartieron a distancia, por medio de la plataforma Zoom.

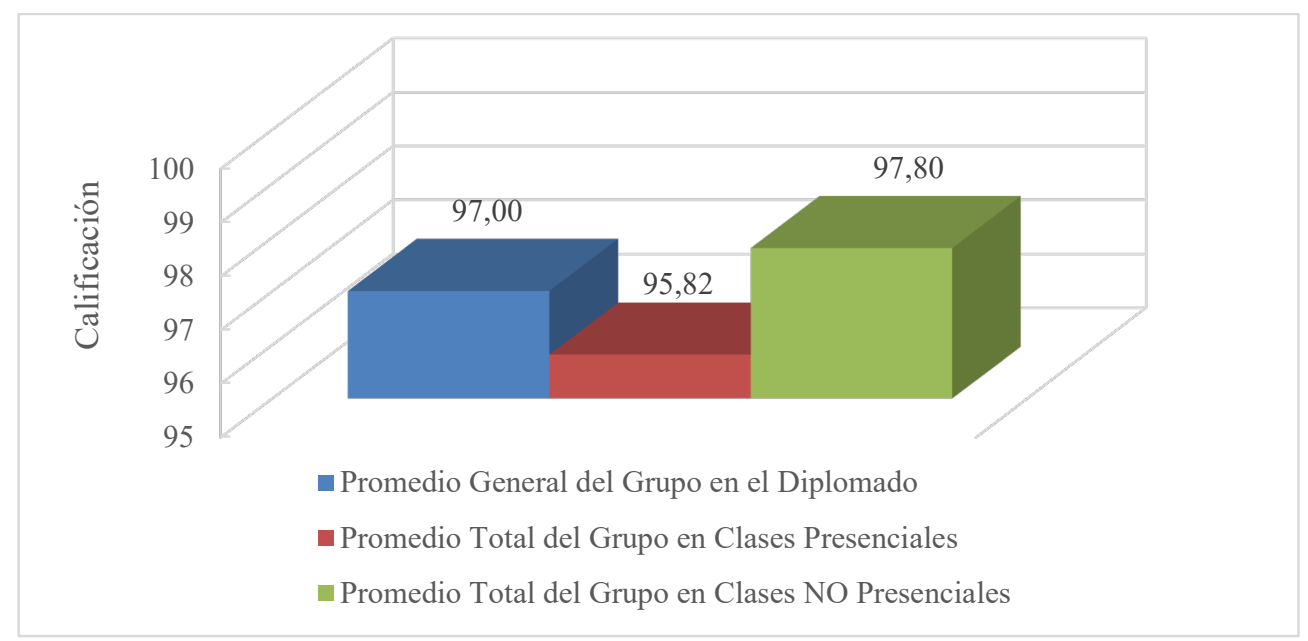

Fig. 8 Comparativa entre las calificaciones finales que se obtuvieron en el diplomado, junto con aquellas clases presenciales y no presenciales.

\section{Conclusiones}

El presente trabajo muestra los resultados obtenidos por el uso de la herramienta Zoom como medio virtual para la transmisión de conocimiento en un caso de estudio sobre un diplomado interinstitucional. Se realizó una comparativa de las notas obtenidas por los alumnos del diplomado que combinó sesiones presenciales y a distancia. Los estudiantes tuvieron mejores notas en las clases a distancia, en donde se obtuvo una nota media de 97,80 en base 100; mientras que las sesiones presenciales obtuvieron una nota media de 95,82 en base 100. La diferencia de 1,98 puntos en nota media entre las sesiones a distancia y las sesiones presenciales está sujeta a diversos factores entre los cuales han de considerarse las dificultades relativas de los diferentes contenidos temáticos entre ambas modalidades de sesión, así como la diversidad de docentes que impartieron los cursos. La diferencia de 1,98 puntos entre las sesiones a distancia y las presenciales podría tomarse como referencia para futuros cursos a distancia, sabiendo que la calificación obtenida por los alumnos estaría desfasada casi dos puntos respecto al mismo curso realizado en modalidad presencial.

La Figura 9 muestra la distribución de las notas promedio tanto para las sesiones nopresenciales como las presenciales. El rango de las mejores notas para las sesiones presenciales estuvo entre 97 a 98 mientras que para las sesiones no-presenciales las mejores notas se encontraron en el rango de 98 a 99. Esta mejora se podría interpretar como que el empleo de Zoom y Moodle como herramientas para sesiones a distancia favorece la nota media de los alumnos. Sin embargo sería necesario evaluar la pertinencia de los medios y 
formas de evaluación tanto para las sesiones a distancia como la presenciales para validar la eficacia directa de Zoom y Moodle.

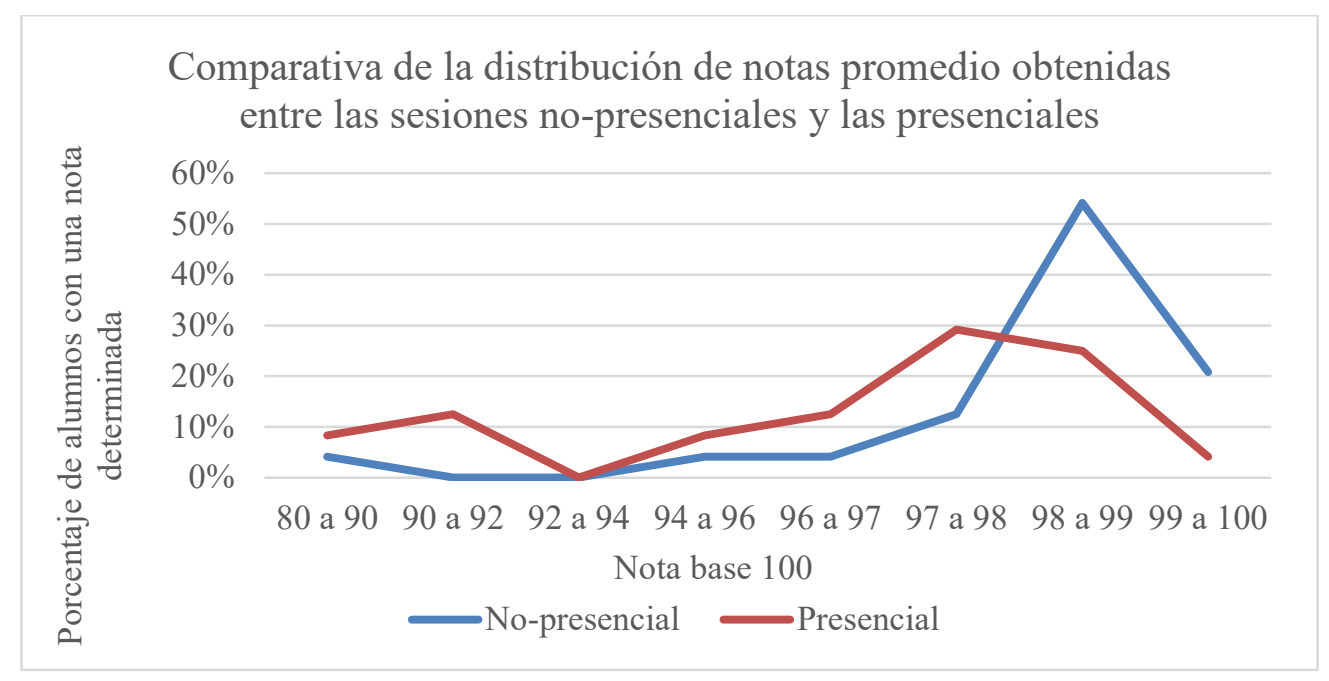

Fig. 9 Distribución de las notas promedio obtenidas por los estudiantes para las sesiones nopresenciales y las presenciales

Una de las posibles causas de mejora en la nota media de los alumnos es que se tuvo la oportunidad de reforzar el aprendizaje al tener la posibilidad de ver y analizar los videos guardados de las conferencias impartidas por el profesor directamente desde Moodle, siendo posible repasar las actividades a cualquier hora y en cualquier lugar con acceso a internet. Habría de evaluarse el efecto de la repetición de la sesión en vídeos grabados versus el esquema tradicional en sesiones presenciales para la mejora en el proceso de evaluación.

Pese a que el esquema presencial tradicional de sesiones ha probado ser efectivo en el desarrollo de habilidades, competencias y adquisición de conocimiento para los alumnos de estudios universitarios, al considerar la mayor cantidad de metodologías disponibles y la mayor facilidad y naturalidad de la interacción alumno-profesor y entre compañeros mismos; el emplear herramientas tecnológicas virtuales como Zoom y Moodle, las cuales pretenden mejorar la interacción alumno-profesor a distancia para hacerla más natural, permite llevar a cabo cursos interinstitucionales e internacionales, donde no siempre es posible una interacción personal de manera presencial, para así ampliar el área de alcance de las universidades, sin que ésta se limite a actividades regionales o nacionales, además de fomentar el intercambio académico y las relaciones internacionales entre universidades o instituciones educativas. 


\section{Referencias}

Blau, I., Weiser, O., \& Eshet-Alkalai, Y. (2016). Face-To-face versus one-way and two-way videoconferencing: How medium naturalness and personality traits influence achievement and perceived learning? Iberian Conference on Information Systems and Technologies, CISTI, 2016-July. https://doi.org/10.1109/CISTI.2016.7521581

De Andrés Martínez, D., Peña Cerdán, A., Ruíz García, J. C., Gisbert Domenech, C., Palomares Chust, A., \& Antonino Daviu, E. (2018). Impulsando el uso de los repositorios institucionales de materiales didácticos multimedia de la UPV. https://doi.org/10.4995/inred2018.2018.8618

Grison, S. (2018). Teaching Toolbox Series : Using " Plickers " to Increase Engagement and Learning What are our goals today?

Heggart, K. R., \& Yoo, J. (2018). Getting the most from google classroom: A pedagogical framework for tertiary educators. Australian Journal of Teacher Education, 43(3), 140-153. https://doi.org/10.14221/ajte.2018v43n3.9

Lenkaitis, C. A. (2019). Technology as a mediating tool: videoconferencing, L2 learning, and learner autonomy. Computer Assisted Language Learning, $0(0)$, 1-27. https://doi.org/10.1080/09588221.2019.1572018

Maher, D., \& Prescott, A. (2017). Professional development for rural and remote teachers using video conferencing. Asia-Pacific Journal of Teacher Education, 45(5), 520-538. https://doi.org/10.1080/1359866X.2017.1296930

Oproiu, G. C. (2015). A Study about Using E-learning Platform (Moodle) in University Teaching Process. Procedia - Social and Behavioral Sciences, 180, 426-432. https://doi.org/10.1016/J.SBSPRO.2015.02.140

Sutterlin, J. (2019). Learning is Social with Zoom Video Conferencing in your Classroom. ELearn Magazine, 1-6.

UPV. (2018). PoliformaT: Formación Online Centro de Formación Permanente. Retrieved March 6, 2019, from https://formaciononline.blogs.upv.es/poliformat-2/poliformat/

Vesisenaho, M., Valtonen, T., Wulff, A., \& Kuittinen, E. (2019). Using Video Conferencing and Video Recordings for Upper Secondary Distance Teaching: Teachers' View Points. In INTED 2016 Proceedings : 10th International Technology, Education and Development Conference (pp. 8582-8589). Retrieved from https://jyx.jyu.fi/bitstream/handle/123456789/49210/vesisenahovaltonenwulffkuittine nusingvideo.pdf? sequence $=1 \&$ isAllowed $=\mathrm{y}$ 